\title{
Biological Evaluation of Newly Synthesized Biaryl Guanidine Derivatives to Arrest $\beta$-Secretase Enzymatic Activity Involved in Alzheimer's Disease
}

\author{
Sayyad Ali, ${ }^{1,2}$ Muhammad Hassham Hassan Bin Asad (D, 1,3 Fahad Khan, \\ Ghulam Murtaza $\mathbb{D}^{5},{ }^{5}$ Albert A. Rizvanov $\mathbb{D}^{\mathrm{D}}{ }^{3}$ Jamshed Iqbal, ${ }^{1}$ Borhan Babak, \\ and Izhar Hussain $\mathbb{D}^{1}$ \\ ${ }^{1}$ Department of Pharmacy, COMSATS University Islamabad, Abbottabad Campus, 22060, Pakistan \\ ${ }^{2}$ Department of Chemistry, Michigan State University, East Lansing, Michigan 48824, USA \\ ${ }^{3}$ Department of Genetics, Institute of Fundamental Medicine and Biology, Kazan Federal University, 420021, Russia \\ ${ }^{4}$ School of Packaging, Michigan State University, East Lansing, Michigan 48824-1223, USA \\ ${ }^{5}$ Department of Pharmacy, COMSATS University Islamabad, Lahore Campus, Pakistan
}

Correspondence should be addressed to Muhammad Hassham Hassan Bin Asad; hasshamasad@yahoo.com and Izhar Hussain; izharhussain@ciit.net.pk

Received 29 June 2019; Revised 20 March 2020; Accepted 17 April 2020; Published 11 May 2020

Academic Editor: Hugo Gutiérrez-De-Terán

Copyright (C) 2020 Sayyad Ali et al. This is an open access article distributed under the Creative Commons Attribution License, which permits unrestricted use, distribution, and reproduction in any medium, provided the original work is properly cited.

\begin{abstract}
Proteases BACE1 ( $\beta$-secretases) enzymes have been recognized as a promising target associated with Alzheimer's disease (AD). This study was carried out on the principles of molecular docking, chemical synthesis, and enzymatic inhibition of BACE1 enzymes via biaryl guanidine-based ligands. Based on virtual screening, thirteen different compounds were synthesized and subsequently evaluated via in vitro and in vivo studies. Among them, 1,3-bis(5,6-difluoropyridin-3-yl)guanidine (compound (9)) was found the most potent $\left(\mathrm{IC}_{50}=97 \pm 0.91 \mathrm{nM}\right.$ ) and active to arrest $(99 \%) \beta$-secretase enzymes (FRET assay). Furthermore, it was found to improve the novel object recognition test and Morris water maze test significantly $(p<0.05)$. Improved pharmacokinetic parameters, viz., $\log P_{\mathrm{o} / \mathrm{w}}(1.76), \log S(-2.73)$, and better penetration to the brain (BBB permeation) with zero Lipinski violation, made it possible to hit the BACE1 as a potential therapeutic source for $\mathrm{AD}$.
\end{abstract}

\section{Introduction}

Alzheimer's disease $(\mathrm{AD})$ is a continuous neurodegenerative disorder that leads to mental deterioration particularly in geriatric population. It is characterized by serious loss of cognition and social and psychiatric anomalies $[1,2]$. Amyloid- $\beta(\mathrm{A} \beta)$ peptide deposition and agglutination of tau proteins are the main pathologic features of the disease that led to the inflammation and eventually loss of neurons $[1,2]$. Indeed, $A \beta$ peptide accumulation resulted from degradation of $\beta$-amyloid precursor proteins (APP) via $\beta$ and $\gamma$ secretase enzymes. The beta site APP cleaving enzyme (BACE1) (composed of 501 extracellular and 22 cytoplasmic amino acids domains) is a main player of producing $\mathrm{A} \beta$ plaques and a promising inhibiting target to control $\mathrm{AD}$. The biological inhibition of BACE1 was focused to inhibit $\mathrm{A} \beta$ formation [2-4].

Statin-type structures were developed initially to lock the two aspartic acids in the catalytic domain; however, these ligands showed very low penetration into the brain. Later on, cyclic and rigid conformational structures (heterocyclic nucleus for enzyme inhibition) were recognized to improve blood-brain barrier (BBB) circulation [5, 6]. To the best of our knowledge, guanidine derivatives have been documented previously to antagonize nervous disorders, cited by Gerritz et al. as acyl guanidines $[6,7]$. We substituted the "acyl" portion with biaryl moieties to find out its inhibition results on 
BACE1. The study was designed to synthesize various symmetrical biaryl guanidine derivatives with particular interest to incorporate fluorines or $-\mathrm{CF}_{3}$ moieties along with heterocyclic rings to the parent nucleus. The in silico study allows a noncovalent bonding interaction of the proposed fluorine and amide $(-\mathrm{NH})$ group within the active site of BACE1 (Asp228 and Asp32); it extends to the Thr231, Tyr71, and Tyr 198 sites. Consequently, we proposed to insert substitutes that could cover the interaction in the S3 pocket. These results gave us a small library of ligands linked to numerous electron-donating and electron-withdrawing groups. These were chemically synthesized to analyze the outcome for BACE1 inhibition [8].

\section{Material and Methods}

2.1. In Silico Study. The in silico study was performed by utilizing the AutoDock Vina program. The X-ray crystallographic structure of BACE1 enzymes (PDB ID: 1FKN, $1.9 \AA$ ) was adopted from the Protein Data Bank (http:// www.rcsb.org) [5, 9]. The addition of water, charges, and hydrogen atoms along with the removal of cocrystallized ligand-protein structures was prepared for molecular docking. The active site was specified via grids around the cocrystallized ligands before the removal of ligands. Gasteiger charges and polar hydrogen were contributed while steady charges were produced using MGL Tools (v 1.5.4). Proposed compounds were sketched and optimized with the ChemDraw Ultra (v 16.0.1, CambridgeSoft Corporation, USA) and MM2 ChemBioDraw software. A grid box (1.0 $)$ was selected to cover up the catalytic site of an enzyme while coordinate centers were marked as 18.439, 4.370, and 9.75. The rest of all necessary parameters were recorded via an online server http://www.swissadme.ch [10].

BACE1 allosteric domains, grid box size $(1.0 \AA)$, and coordinates were specified as 50,48, and 52. Moreover, nine poses (generated via the Lamarckian Genetic Algorithm based on binding free energies) were fixed and visualized with the PyMol molecular viewer (v 2.1, Schrödinger Inc., New York, NY, USA) and Discovery Studio (v 17.2, BIOVIA Corp., San Diego, CA, USA) software [11]. Ligand-enzyme interaction and various pharmacokinetic properties, i.e., $\mathrm{Clog} P$, Lipinski, and BBB permeation, were obtained via an online server http://www.swissadme.ch [10]. All the proposed ligands were synthesized after a detailed in silico authentication.

2.2. Chemical Synthesis (N,N'-Biaryl Guanidines). N, $N^{\prime}$ -Biaryl guanidine derivatives were synthesized via arylation of guanidine moieties according to the methods described by Hui et al. [12].

Briefly, Schlenk line flask mixture was composed of guanidine nitrate $(1.0 \mathrm{mmol})$, aryl halides $(1.0 \mathrm{mmol}$, Ar$\mathrm{I} / \mathrm{Br})$, ligand $\mathrm{N}$-methylglycine $(8.9 \mathrm{mg} / 0.2 \mathrm{mmol}$ for Ar-I; $17.8 \mathrm{mg} / 0.1 \mathrm{mmol}$ for $\mathrm{Ar}-\mathrm{Br})$, recrystallized $\mathrm{CuI}$ (9.5 mg/0.05 mmol for Ar-I; $19 \mathrm{mg} / 0.1 \mathrm{mmol}$ for Ar-Br), and $\mathrm{K}_{3} \mathrm{PO}_{4}(1.270 \mathrm{~g} / 6 \mathrm{mmol})$ under described reaction conditions. Reaction mixture was evacuated and backfilled, and acetonitrile $(5 \mathrm{ml})$ was added subsequently. It was continu- ously stirred until aryl halide was completely utilized (monitored periodically with TLC) and collected through ethyl acetate. Brine solution was added after separation of the upper organic layer and dried with $\mathrm{Na}_{2} \mathrm{SO}_{4}$. Furthermore, the material was purified through silica gel column chromatography with eluents methanol/methylene chloride in the ratio of 25:1-40:1 to acquire the final product(s).

2.3. Spectral Analysis and Other Parameters. The ${ }^{1} \mathrm{H}-\mathrm{NMR}$, ${ }^{13} \mathrm{C}-\mathrm{NMR}$, and ${ }^{19} \mathrm{~F}-\mathrm{NMR}$ spectra were recorded by Agilent (DDR2 $500 \mathrm{MHz}$ NMR spectrometers) equipped with 7600AS 96 sample autosamplers running VnmrJ 3.2A. TMS was used as the internal standard while the chemical shift and coupling constant $(J)$ were described in ppm $(\delta)$ and $\mathrm{MHz}$, respectively. Other related information of the synthesized ligands (Clog $P$, Lipinski, BBB permeation, and pharmacokinetics) was obtained from an online server http://www .swissadme.ch/.

2.4. Fluorescence Resonance Energy Transfer (FRET) Assay. BACE1 inhibitory activity was carried out by adopting the procedures described by the Sigma-Aldrich FRET-based assay activity kit (product \# CS0010).

Briefly, the assay was carried out in a fixed volume of $100 \mu \mathrm{l}$ with the BACE1 substrate $(20 \mu \mathrm{l}, 50 \mu \mathrm{M})$, assay buffer (78 $\mu \mathrm{l} / 78-X \mu \mathrm{l}, \mathrm{pH} 4.5)$, test sample(s) $(X \mu \mathrm{l}, 100 \mu \mathrm{M})$, and BACE1 enzyme $(2 \mu \mathrm{l}, \sim 0.3 \mathrm{unit} / \mu \mathrm{l})$ incubated at $37^{\circ} \mathrm{C}$ for $75 \mathrm{~min}$ in 96-well microplate reaction mixture [13]. The baseline fluorescence (null time) signal was noticed (at $320 \mathrm{~nm}$ ) immediately after the addition of BACE1 enzymes while emission signals were monitored at $405 \mathrm{~nm}$ at $25^{\circ} \mathrm{C}$. Both the enzyme and the substrate were prepared in the buffer while tested samples dissolved primarily in DMSO (5\%) subsequently in serially diluted buffer solution in a desired volume of $2 \mu \mathrm{l}, 3 \mu \mathrm{l}$, and $5 \mu \mathrm{l}$ having $200 \mathrm{pmol}$, $300 \mathrm{pmol}$, and $500 \mathrm{pmol}$ concentration, respectively. A standard curve was prepared between fluorescent unit (FU) and standard solution $(100 \mu \mathrm{M}, 100-500 \mathrm{pmol}$, and 1-5 $\mu \mathrm{l})$ to find out $50 \%$ BACE1 cleaving activity [14]. The sample blank (buffer with substrate) was treated as the negative control while the positive control was the solution of the buffer with substrate and enzyme mixture (http://www.sigmaaldrich .com). Finally, $\mathrm{IC}_{50}$ of the intended compounds were calculated via GraphPad Prism v8 software.

2.5. Experimental Animals. Male BALB/c mice (3.5 to 5.5 months of age) were purchased from National Institute of Health (NIH) Islamabad, Pakistan. All experiments were performed by following the rules and regulations in accordance with the Helsinki Declaration after getting permission from the Departmental Review Committee (Ref. \# PHM.Eth/CS-M01/18-001 dated 05/2018). Animals were kept in standard cages and provided food, water, and cleanliness in accordance with the documented protocols. Moreover, they were categorized into nine groups $(n=10)$ for experimental purposes.

2.6. Animal Preparation. The control group of mice was treated with drinking water having aluminum chloride $(17 \mathrm{mg} / \mathrm{kg})$ and tape water for a period of five weeks. 

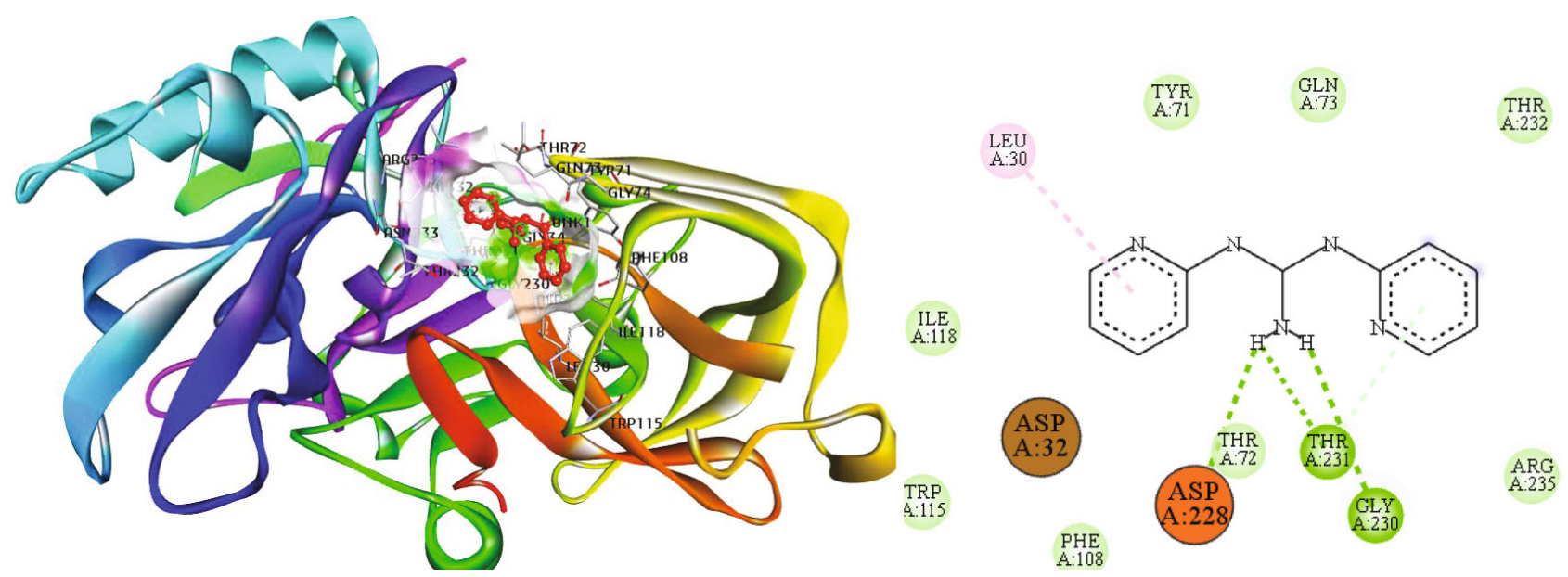

(a) Compound (5) interactions within the flap conformation of BACE1
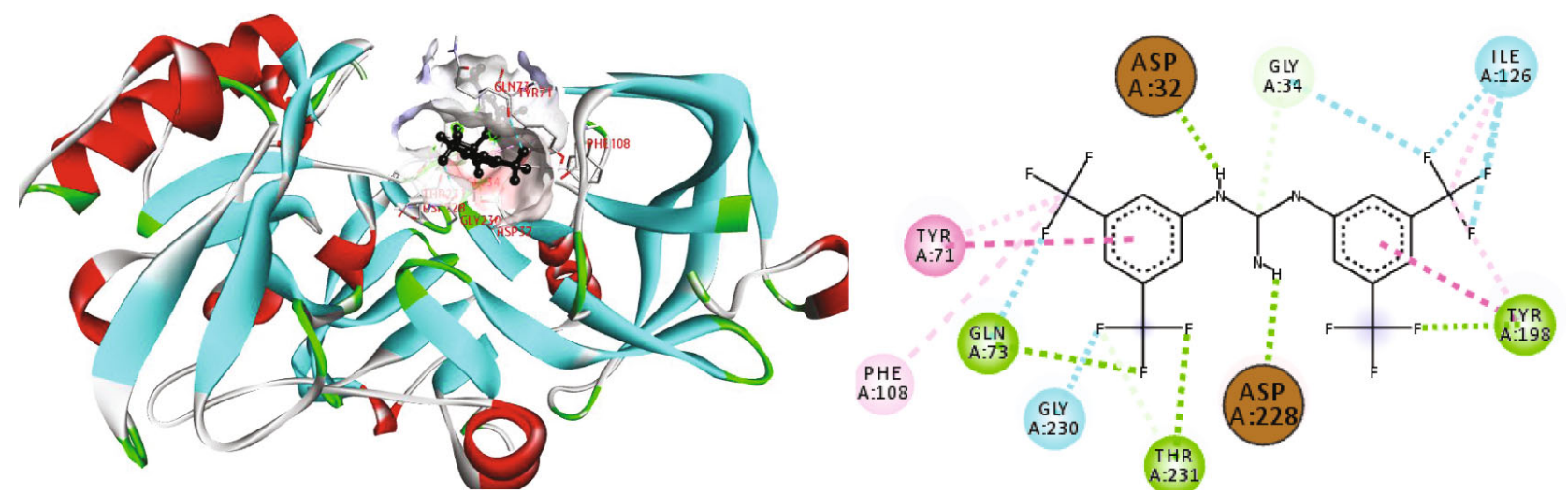

(b) The molecular interactions of compound (8) within the active domain of BACE1
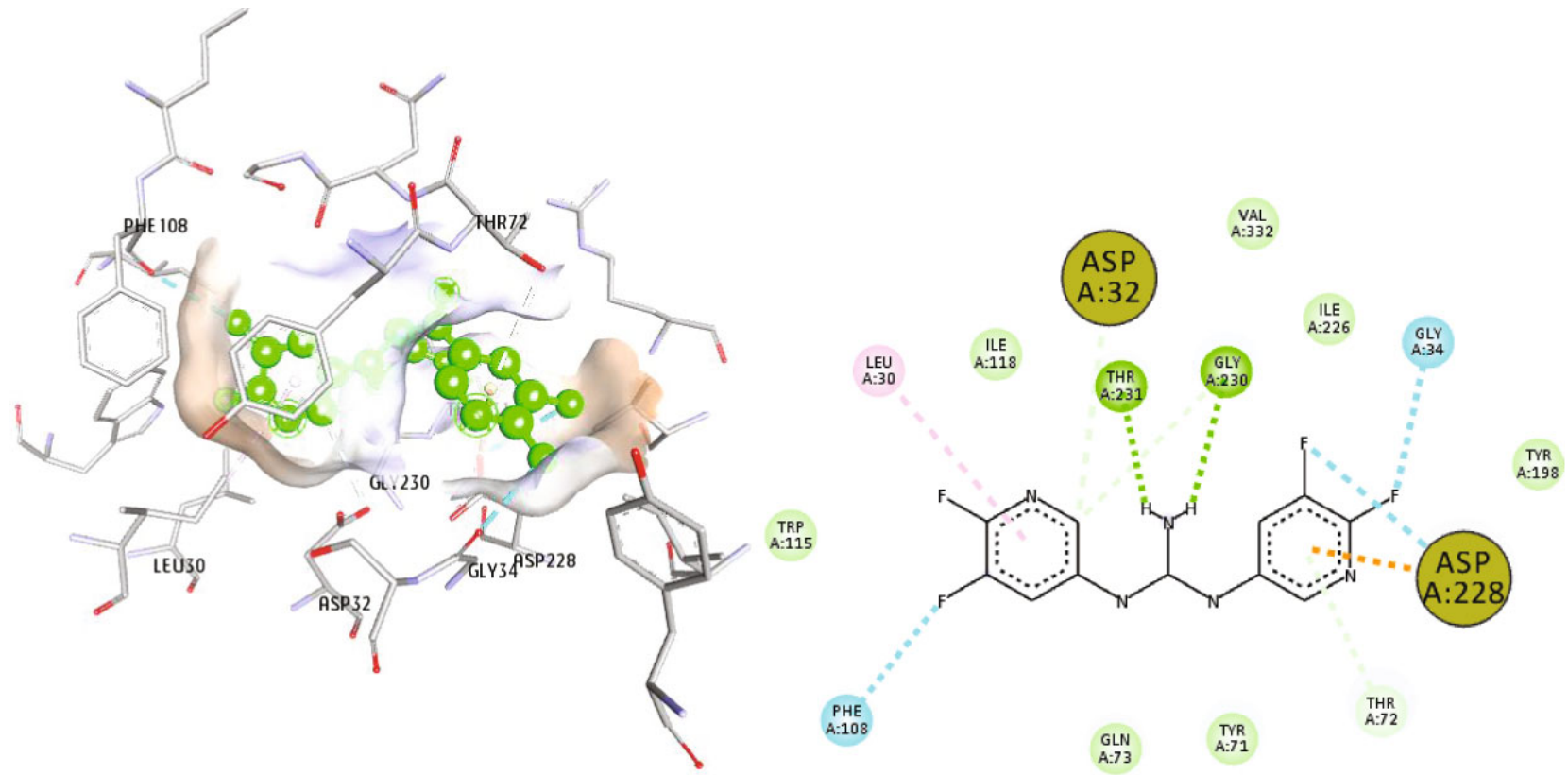

(c) Compound (9)

FIGURE 1: The possible interaction of compounds (5), (8), and (9) within the active domain of BACE1 that completely arrested the catalytic activity of aspartate residues for this enzyme in a closed conformation of the flap region. 
TABLE 1: Detailed information about newly synthesized N,N' -biaryl guanidine derivatives to arrest BACE1 enzymatic activity.

\begin{tabular}{|c|c|c|c|}
\hline Code & $\begin{array}{l}\text { Structure of the compound } \\
\text { with name }\end{array}$ & Spectral detail (NMR \& ESI-MS) & Miscellaneous features \\
\hline 1 & $\begin{array}{c}\text { 1,3-Bis (2-fluorophenyl) } \\
\text { guanidine }\end{array}$ & $\begin{array}{c}{ }^{1} \mathrm{H}-\mathrm{NMR}\left(500 \mathrm{MHz}, \mathrm{CD}_{3} \mathrm{OD}\right): \delta 8.6(2 \mathrm{H}, \mathrm{NH}, \mathrm{s}), \\
8.2(1 \mathrm{H}, \mathrm{NH}, \mathrm{s}), 7.3(2 \mathrm{H}, \mathrm{d}, J=7.6 \mathrm{~Hz}), 7.0(2 \mathrm{H}, \\
\mathrm{d}, J=7.5 \mathrm{~Hz}), 6.8(2 \mathrm{H}, \mathrm{d}, J=9 \mathrm{~Hz}), 6.7(2 \mathrm{H}, \mathrm{d}, \\
J=7.5 \mathrm{~Hz}) ;{ }^{13} \mathrm{C}-\mathrm{NMR}\left(125 \mathrm{MHz}, \mathrm{CD}_{3} \mathrm{OD}\right): \delta \\
165.3,163.3,139.5(2 \mathrm{C}), 135.7,132.0(2 \mathrm{C}), 122.7 \\
(2 \mathrm{C}), 122.6(2 \mathrm{C}), 114.1,113.9 ;{ }^{19} \mathrm{~F}-\mathrm{NMR} \\
\left(470 \mathrm{MHz}, \mathrm{CD}_{3} \mathrm{OD}\right): \delta-108.06,-125.11 ; \mathrm{HR}-\mathrm{EI} \\
\mathrm{MS}: m / z 247.09201 ;\left[(M+1){ }^{+} \text {calculated for }\right. \\
\left.\mathrm{C}_{13} \mathrm{H}_{11} \mathrm{~F}_{2} \mathrm{~N}_{3} 247.09210\right] . \\
\end{array}$ & $\begin{array}{l}55 \% \text { yield; white solid; } \mathrm{mp} 130-132^{\circ} \mathrm{C} ; \mathrm{IC}_{50}=85 \\
\pm 1.84 \mu \mathrm{M} \text { HB_donor } 3 ; \mathrm{HB} \_ \text {acceptor } 3 \\
\text { rotatable bonds } 4 ; \mathrm{Ar}-\mathrm{Br} \text { was used to synthesize; } \\
\mathrm{M} . \mathrm{Wt}=247.092 \mathrm{~g} / \mathrm{mol}\end{array}$ \\
\hline 2 & $\begin{array}{l}\text { 1,3-Bis (4-bromonaphtalen-1-yl) } \\
\text { guanidine }\end{array}$ & $\begin{array}{c}{ }^{1} \mathrm{H}-\mathrm{NMR}\left(500 \mathrm{MHz}, \mathrm{CD}_{3} \mathrm{OD}\right): \delta 8.8(2 \mathrm{H}, \mathrm{NH}, \mathrm{s}), \\
8.2(2 \mathrm{H}, \mathrm{d}, J=7.8 \mathrm{~Hz}), 7.9(2 \mathrm{H}, \mathrm{d}, J=7.1 \mathrm{~Hz}), 7.8 \\
(3 \mathrm{H}, \mathrm{d}, J=8.1 \mathrm{~Hz}), 7.4(4 \mathrm{H}, \mathrm{m}), 6.9(2 \mathrm{H}, \mathrm{d}, J=7 \\
\mathrm{Hz}) ;{ }^{13} \mathrm{C}-\mathrm{NMR}\left(125 \mathrm{MHz}, \mathrm{CD}_{3} \mathrm{OD}\right): \delta 161.3, \\
143.2(2 \mathrm{C}), 131.9(2 \mathrm{C}), 131.1(2 \mathrm{C}), 129.3(2 \mathrm{C}), \\
128.7(2 \mathrm{C}), 126.8(2 \mathrm{C}), 126.3(2 \mathrm{C}), 121.6(2 \mathrm{C}), \\
114.5(2 \mathrm{C}), 110.9(2 \mathrm{C}) ; \mathrm{HR}-\mathrm{EI} \mathrm{MS:} \mathrm{m} / z \\
466.96318 ;\left[(M+1)^{+} \text {calculated for }\right. \\
\left.\mathrm{C}_{21} \mathrm{H}_{15} \mathrm{Br}_{2} \mathrm{~N}_{3} 466.96327\right] .\end{array}$ & $\begin{array}{c}79 \% \text { yield; white solid; mp } 128-130^{\circ} \mathrm{C} ; \mathrm{IC}_{50}= \\
235 \pm 1.90 \mu \mathrm{M} ; \mathrm{HB} \text { _donor } 4 ; \mathrm{HB} \text { acceptor } 1 \\
\text { rotatable bonds } 3 ; \mathrm{Ar}-\mathrm{Br} \text { was used to synthesize; } \\
\mathrm{M} . \mathrm{Wt}=466.963 \mathrm{~g} / \mathrm{mol}\end{array}$ \\
\hline
\end{tabular}

3

1,3-Bis (2-nitrophenyl) guanidine
${ }^{1} \mathrm{H}-\mathrm{NMR}\left(500 \mathrm{MHz}, \mathrm{CD}_{3} \mathrm{OD}\right): \delta 8.6(2 \mathrm{H}, \mathrm{NH}, \mathrm{s})$, $8.3(1 \mathrm{H}, \mathrm{NH}, \mathrm{s}), 7.7(3 \mathrm{H}, \mathrm{m}), 7.4(3 \mathrm{H}, \mathrm{m}), 7.0$ $(2 \mathrm{H}, \mathrm{m}) ;{ }^{13} \mathrm{C}-\mathrm{NMR}\left(125 \mathrm{MHz}, \mathrm{CD}_{3} \mathrm{OD}\right): \delta 158.5$, 139.7 (2C), 132.5 (2C), 131.0 (2C), 126.4 (2C), 123.0 (2C), 111.0 (2C); HREI MS: $m / z$ 301.8121; $\left[(M+1)^{+}\right.$calculated for $\left.\mathrm{C}_{13} \mathrm{H}_{11} \mathrm{~N}_{5} \mathrm{O}_{4} 301.8110\right]$.
$92 \%$ yield; yellow solid; $\mathrm{mp} 136-138^{\circ} \mathrm{C}$; $\mathrm{IC}_{50}=$ $259 \pm 2.50 \mu \mathrm{M} ; \mathrm{HB} \_$donor 5; HB_acceptor 3; rotatable bonds 6; Ar-Br was used to synthesize; $\mathrm{M} . \mathrm{Wt}=301.881 \mathrm{~g} / \mathrm{mol}$<smiles>N=C(Nc1ccc2ncccc2c1)Nc1ccc2ncccc2c1</smiles>

${ }^{1} \mathrm{H}-\mathrm{NMR}\left(500 \mathrm{MHz}, \mathrm{CD}_{3} \mathrm{OD}\right): \delta 8.2(2 \mathrm{H}, \mathrm{s}), 7.6$ $(3 \mathrm{H}, \mathrm{d}, J=7.5 \mathrm{~Hz}), 6.9(3 \mathrm{H}, \mathrm{t}, J=7.8 \mathrm{~Hz}) ;{ }^{13} \mathrm{C}-$ NMR (125 MHz, $\left.\mathrm{CD}_{3} \mathrm{OD}\right): \delta 160.8,159.8(2 \mathrm{C})$, 149.2 (2C), 139.7 (2C), 119.4 (2), 111.9 (2C); HREI MS: $m / z$ 213.10133; $\left[(M+1)^{+}\right.$calculated for $\mathrm{C}_{11} \mathrm{H}_{11} \mathrm{~N}_{5}$ 213.10145].
$88 \%$ yield; white solid; $\mathrm{mp} 140-142^{\circ} \mathrm{C} ; \mathrm{IC}_{50}=9$ $\pm 0.05 \mu \mathrm{M}$; HB_donor 3; HB_acceptor 3; rotatable bonds 4; $\mathrm{Ar}-\mathrm{Br}$ was used to synthesize; $\mathrm{M} . \mathrm{Wt}=213.101 \mathrm{~g} / \mathrm{mol}$

${ }^{1} \mathrm{H}-\mathrm{NMR}\left(500 \mathrm{MHz}, \mathrm{CD}_{3} \mathrm{OD}\right): \delta 9.8(1 \mathrm{H}, \mathrm{NH}, \mathrm{s})$, $8.6(2 \mathrm{H}, \mathrm{d}, J=8 \mathrm{~Hz}), 8.3(1 \mathrm{H}, \mathrm{NH}, \mathrm{s}), 8.1(1 \mathrm{H}$, $\mathrm{NH}, \mathrm{S}), 8.0(1 \mathrm{H}, \mathrm{s}), 7.7(1 \mathrm{H}, \mathrm{s}), 7.5(2 \mathrm{H}, \mathrm{d}, J=5)$, $7.3(6 \mathrm{H}, \mathrm{m}) .{ }^{13} \mathrm{C}-\mathrm{NMR}\left(125 \mathrm{MHz}, \mathrm{CD}_{3} \mathrm{OD}\right): \delta$ 161.6, 146 (2C), 143.2 (2C), 138.3 (2C), 133.4 (2C), 132.6 (2C), 130.3 (2C), 124.9 (2C), 123.5 (2C), 121.7 (2C); HREI MS: $m / z$ 313.13265; $\left[(M+1)^{+}\right.$calculated for $\left.\mathrm{C}_{19} \mathrm{H}_{15} \mathrm{~N}_{5} 313.13275\right]$.

$90 \%$ yield; white solid; $\mathrm{mp} 133-134^{\circ} \mathrm{C} ; \mathrm{IC}_{50}=91$ $\pm 0.19 \mathrm{nM}$; HB_donor 3; HB_acceptor 3; rotatable bonds 4; $\mathrm{Ar}-\mathrm{Br}$ was used to synthesize; $\mathrm{M} . \mathrm{Wt}=313.132 \mathrm{~g} / \mathrm{mol}$ ${ }^{1} \mathrm{H}-\mathrm{NMR}\left(500 \mathrm{MHz}, \mathrm{CD}_{3} \mathrm{OD}\right): \delta 9.2(2 \mathrm{H}, \mathrm{NH}, \mathrm{s})$, $8.2(2 \mathrm{H}, \mathrm{m}), 7.9(2 \mathrm{H}, \mathrm{d}, J=8.5 \mathrm{~Hz}), 7.8(2 \mathrm{H}, \mathrm{m})$, $7.6(1 \mathrm{H}, \mathrm{NH}, \mathrm{S}), 7.5(2 \mathrm{H}, \mathrm{dt}, J=7 \mathrm{~Hz}), 7.4(1 \mathrm{H}$, m), $7.2(2 \mathrm{H}, \mathrm{m}), 6.8(1 \mathrm{H}, \mathrm{S}) ;{ }^{13} \mathrm{C}-\mathrm{NMR}$ (125 MHz, $\left.\mathrm{CD}_{3} \mathrm{OD}\right): \delta$ 161.5, 160.4 (2C), 139.3 (2C), 134.5 (2C), 129.3 (2C), 127.6 (2C), 127.1 (2C), 124.6 (2C), 123.1 (2C), 118.0 (2C) 105.6 (2C); ${ }^{19} \mathrm{~F}-\mathrm{NMR}\left(470 \mathrm{MHz}, \mathrm{CD}_{3} \mathrm{OD}\right): \delta$-75.2;

$63 \%$ yield; white solid; $\mathrm{mp} 126-128^{\circ} \mathrm{C}$; $\mathrm{IC}_{50}=$ $102 \pm 0.90$ nM; HB_donor 3; HB_acceptor 3; rotatable bonds 4; Ar-Br was used to synthesize; $\mathrm{M} . \mathrm{Wt}=347.123 \mathrm{~g} / \mathrm{mol}$ for $\mathrm{C}_{21} \mathrm{H}_{15} \mathrm{~F}_{2} \mathrm{~N}_{3}$ 347.12340]. 
TABle 1: Continued.

\begin{tabular}{|c|c|c|c|}
\hline Code & $\begin{array}{l}\text { Structure of the compound } \\
\text { with name }\end{array}$ & Spectral detail (NMR \& ESI-MS) & Miscellaneous features \\
\hline 7 & $\begin{array}{l}\text { 1,3-Di (naphthalen-2-yl) } \\
\text { guanidine }\end{array}$ & $\begin{array}{c}{ }^{1} \mathrm{H}-\mathrm{NMR}\left(500 \mathrm{MHz}, \mathrm{CD}_{3} \mathrm{OD}\right): \delta 9.2(2 \mathrm{H}, \mathrm{NH}, \mathrm{s}), \\
8.2(4 \mathrm{H}, \mathrm{m}), 7.94(1 \mathrm{H}, \mathrm{q}, J=5,5.5 \mathrm{~Hz}), 7.8(2 \mathrm{H}, \\
\mathrm{tt}, J=7,7.5 \mathrm{~Hz}), 7.8(3 \mathrm{H}, \mathrm{m}), 7.4(2 \mathrm{H}, \mathrm{q}, J=8.5 \\
\mathrm{Hz}), 6.8(1 \mathrm{H}, \mathrm{NH}, \mathrm{s}) ;{ }^{13} \mathrm{C}-\mathrm{NMR}(125 \mathrm{MHz}, \\
\left.\mathrm{CD}_{3} \mathrm{OD}\right): \delta 161.9,137.5(2 \mathrm{C}), 135.2(4 \mathrm{C}), 134.5 \\
(2 \mathrm{C}), 133.2(2 \mathrm{C}), 132.0(2 \mathrm{C}), 129.5(2 \mathrm{C}), 126.1 \\
(2 \mathrm{C}), 121.7(2 \mathrm{C}), 116.4(2 \mathrm{C}) ; \mathrm{HREI} \mathrm{MS}: \mathrm{m} / z \\
311.14215 ;\left[(M+1)^{+} \text {calculated for } \mathrm{C}_{21} \mathrm{H}_{17} \mathrm{~N}_{3}\right. \\
311.14225] .\end{array}$ & $\begin{array}{c}91 \% \text { yield; white solid; } \mathrm{mp} 146-148^{\circ} \mathrm{C} ; \mathrm{IC}_{50}= \\
159 \pm 1.10 \mu \mathrm{M} ; \mathrm{HB} \text { _donor } 3 ; \mathrm{HB} \text { acceptor } 1 \\
\text { rotatable bonds } 4 ; \mathrm{Ar}-\mathrm{Br} \text { was used to synthesize; } \\
\mathrm{M} . \mathrm{Wt}=311.142 \mathrm{~g} / \mathrm{mol}\end{array}$ \\
\hline 8 & $\underset{\text { 1,3-Bis (3,5-bis (trifluoromethyl) }}{\text { phenyl) guanidine }}$ & $\begin{array}{c}{ }^{1} \mathrm{H}-\mathrm{NMR}\left(500 \mathrm{MHz}, \mathrm{CD}_{3} \mathrm{OD}\right): \delta 8.9(2 \mathrm{H}, \mathrm{NH}, \mathrm{s}) \text {, } \\
7.3(3 \mathrm{H}, \mathrm{s}), 5.7(4 \mathrm{H}, \mathrm{s}) ;{ }^{13} \mathrm{C}-\mathrm{NMR}(125 \mathrm{MHz}, \\
\left.\mathrm{CD}_{3} \mathrm{OD}\right): \delta 161.3,146.3(2 \mathrm{C}), 134.7(4 \mathrm{C}, \mathrm{d}, J=5 \\
.1 \mathrm{~Hz}), 131.4(2 \mathrm{C}), 125.8(4 \mathrm{C}, \mathrm{d}, J=271.3 \mathrm{~Hz}) \\
123.7(2 \mathrm{C}, \mathrm{d}, J=34.6 \mathrm{~Hz}), 115.7(2 \mathrm{C}) ;{ }^{19} \mathrm{~F}-\mathrm{NMR} \\
\left(470 \mathrm{MHz}, \mathrm{CD}_{3} \mathrm{OD}\right): \delta-61.8,-61.5 ; \mathrm{HREI} \mathrm{MS}: \\
m / z 483.06039 ;\left[(M+1){ }^{+} \text {calculated for }\right. \\
\left.\mathrm{C}_{17} \mathrm{H}_{9} \mathrm{~F}_{12} \mathrm{~N}_{3} 483.06049\right] .\end{array}$ & $\begin{array}{l}68 \% \text { yield; yellowish solid; } \mathrm{mp} 76-78^{\circ} \mathrm{C} ; \mathrm{IC}_{50}= \\
229 \pm 2.10 \mu \mathrm{M} ; \mathrm{HB} \text { _donor } 3 ; \mathrm{HB} \text { _acceptor } 13 \\
\text { rotatable bonds } 8 ; \text { Ar-I was used to synthesize; } \\
\quad \mathrm{M} . \mathrm{Wt}=483.060 \mathrm{~g} / \mathrm{mol}\end{array}$ \\
\hline
\end{tabular}<smiles>N=C(Nc1cnc(F)c(F)c1)Nc1cnc(F)c(F)c1</smiles>

1,3-Bis (5,6-difluoropyridin-3-yl) guanidine
${ }^{1} \mathrm{H}-\mathrm{NMR}\left(500 \mathrm{MHz}, \mathrm{CD}_{3} \mathrm{OD}\right): \delta 8.7(2 \mathrm{H}, \mathrm{NH}, \mathrm{s})$, $8.3(2 \mathrm{H}, \mathrm{s}), 7.4(1 \mathrm{H}, \mathrm{s}), 7.3(2 \mathrm{H}, \mathrm{t}, J=10.5 \mathrm{~Hz}) ; \quad 61 \%$ yield, whitish yellow solid; mp $128-130^{\circ} \mathrm{C}$; ${ }^{13} \mathrm{C}$-NMR $\left(125 \mathrm{MHz}, \mathrm{CD}_{3} \mathrm{OD}\right): \delta 166.9(2 \mathrm{C}), \quad \mathrm{IC}_{50}=97 \pm 0.91 \mathrm{nM}$; HB_donor 3; HB_acceptor 158.5, 146.3 (2C), 143.2 (2C), 135.7 (2C), 121.5 (2C); HREI MS: $m / z$ 285.06376; $\left[(M+1)^{+}\right.$ calculated for $\mathrm{C}_{11} \mathrm{H}_{7} \mathrm{~F}_{4} \mathrm{~N}_{5}$ 285.06386].
7; rotatable bonds 4; $\mathrm{Ar}-\mathrm{Br}$ was used to synthesize; M.Wt $=285.063 \mathrm{~g} / \mathrm{mol}$
${ }^{1} \mathrm{H}-\mathrm{NMR}\left(500 \mathrm{MHz}, \mathrm{CD}_{3} \mathrm{OD}\right): \delta 7.5(2 \mathrm{H}, \mathrm{NH}, \mathrm{s})$, $7.3(4 \mathrm{H}, \mathrm{d}, J=8.5 \mathrm{~Hz}), 7.3(4 \mathrm{H}, \mathrm{d}, J=8.5 \mathrm{~Hz}), 6.7$ $(1 \mathrm{H}, \mathrm{NH}, \mathrm{s}) ;{ }^{13} \mathrm{C}-\mathrm{NMR}\left(125 \mathrm{MHz}, \mathrm{CD}_{3} \mathrm{OD}\right): \delta$ 163.3, 153.4, 133.8 (4C), 121.1 (4C); HREI MS: $\mathrm{m} / \mathrm{z}$ 368.93187; $\left[(M+1)^{+}\right.$calculated for $\mathrm{C}_{13} \mathrm{H}_{11} \mathrm{Br}_{2} \mathrm{~N}_{3}$ 368.93197].
$72 \%$ yield; white solid; $\mathrm{mp} 250-252^{\circ} \mathrm{C}$; $\mathrm{IC}_{50}=$ $321 \pm 2.15 \mu \mathrm{M}$; HB_donor 3; HB_acceptor 1 ; rotatable bonds 4; Ar-Br was used to synthesize; $\mathrm{M} . \mathrm{Wt}=368.931 \mathrm{~g} / \mathrm{mol}$

1,3-Bis (4-bromophenyl) guanidine

11<smiles>[NH2+]=C(Nc1ccc(Cl)cc1F)Nc1ccc(Cl)cc1F</smiles>

1,3-Bis (4-chloro-2-fluorophenyl) guanidine
${ }^{1} \mathrm{H}-\mathrm{NMR}\left(500 \mathrm{MHz}, \mathrm{CD}_{3} \mathrm{OD}\right): \delta 8.4(2 \mathrm{H}, \mathrm{NH}, \mathrm{s})$ $7.9(2 \mathrm{H}, \mathrm{dd}, J=2,10.5 \mathrm{~Hz}), 7.8(2 \mathrm{H}, \mathrm{d}, J=7 \mathrm{~Hz})$, $7.6(2 \mathrm{H}, \mathrm{t}, J=9 \mathrm{~Hz}), 6.2(1 \mathrm{H}, \mathrm{s}) ;{ }^{13} \mathrm{C}-\mathrm{NMR}$

(125 MHz, $\left.\mathrm{CD}_{3} \mathrm{OD}\right): \delta 159.1(2 \mathrm{C}), 130.3(2 \mathrm{C})$, 126.2 (2C), 121.2 (2C), HREI MS: $m / z$ 315.01425; $\left[(M+1)^{+}\right.$calculated for $\mathrm{C}_{13} \mathrm{H}_{9} \mathrm{Cl}_{2} \mathrm{~F}_{2} \mathrm{~N}_{3}$ 315.01416].
$69 \%$ yield; white solid; $\mathrm{mp} 160-162^{\circ} \mathrm{C}$; $\mathrm{IC}_{50}=$ $172 \pm 1.50 \mu \mathrm{M}$; HB_donor 3; HB_acceptor 3; rotatable bonds 4; Ar-I was used to synthesize; $\mathrm{M} . \mathrm{Wt}=315.014 \mathrm{~g} / \mathrm{mol}$

\footnotetext{
${ }^{1} \mathrm{H}-\mathrm{NMR}\left(500 \mathrm{MHz}, \mathrm{CD}_{3} \mathrm{OD}\right): \delta 7.7(2 \mathrm{H}, \mathrm{NH}, \mathrm{s})$,<smiles>Fc1ccc(NCNc2ccc(F)cc2)cc1</smiles>

1,3-Bis (4-fluorophenyl) guanidine

${ }^{1} \mathrm{H}-\mathrm{NMR}\left(500 \mathrm{MHz}, \mathrm{CD}_{3} \mathrm{OD}\right): \delta 7.7(2 \mathrm{H}, \mathrm{NH}, \mathrm{s})$,
$7.1(4 \mathrm{H}, \mathrm{t}, J=8 \mathrm{~Hz}), 7.0(5 \mathrm{H}, \mathrm{t}, J=8.5 \mathrm{~Hz}),{ }^{13} \mathrm{C}-$ NMR $\delta\left(125 \mathrm{MHz}, \mathrm{CD}_{3} \mathrm{OD}\right) 161.3,159.47(2 \mathrm{C})$, $127.6(2 \mathrm{C}), 127.1(2 \mathrm{C}), 126.7(2 \mathrm{C}), 121.5(2 \mathrm{C})$, $116.3(2 \mathrm{C}) ;{ }^{19} \mathrm{~F}-\mathrm{NMR}\left(470 \mathrm{MHz}, \mathrm{CD}_{3} \mathrm{OD}\right): \delta$ -123.4, -123.1; HREI MS: $m / z$ 247.11200; $\left[(M+1)^{+}\right.$calculated for $\mathrm{C}_{13} \mathrm{H}_{11} \mathrm{~F}_{2} \mathrm{~N}_{3}$ 247.11210].
}

$71 \%$ yield; white solid; $\mathrm{mp} 146-148^{\circ} \mathrm{C}$; $\mathrm{IC}_{50}=$ $103 \pm 0.95 \mu \mathrm{M}$; HB_donor 3; HB_acceptor 3; rotatable bonds 4; Ar-Br was used to synthesize; M.Wt $=247.112 \mathrm{~g} / \mathrm{mol}$ 
TABLE 1: Continued.

Code $\begin{gathered}\text { Structure of the compound } \\ \text { with name }\end{gathered}$

Note: NMR: nuclear magnetic resonance; ESI-MS: electrospray ionization-mass spectrometry; mp: melting point; HB: hydrogen bonding; M. Wt: molecular weight.

Synthesized ligands (20-55 mg/kg) were administered to neutralize the $\mathrm{AlCl}_{3}$ effects and to design treated mouse groups. All the behavioral tests were performed on days 28 to 34 $\left(08: 30\right.$ am to $4: 30 \mathrm{pm}$ at $\left.25 \pm 2^{\circ} \mathrm{C}\right)$. Animals were shifted 20 minutes prior to the experimentation room to get accustomed with the test environment. Trials were recorded via videocam, and results were obtained to reveal the final conclusions [15-17].

2.7. Morris Water Maze Test. To evaluate 3D spatial recalling memory of the animals under observations, an authentic and sensitive assay "Morris water maze test" was carried out [13].

The apparatus used for this test has a round pool with a $120 \mathrm{~cm}$ diameter and a $60 \mathrm{~cm}$ of depth. The pool is divided into 4 hypothetical quadrants North, West, South, and East. The experiment began on day 28, and 5 trials were executed each day. The mouse was given $1 \mathrm{~min}$ to find out the hidden platform with a $10 \mathrm{~min}$ interval between the two successive trials. The usual time essential for a mouse to reach at the platform was recorded, and an average of 5 trials was presumed as the escape latency of the animal for that day. On the $32^{\text {nd }}$ day of intervention, a probe test was executed when there was no platform, with a different release position. The spatial memory of the mouse was estimated by computing the time spent by the mouse in the quadrant in which the platform was formerly located. The number of crossings across the earlier platform position was also found out $[18,19]$.

2.8. Novel Object Recognition Memory Test. The novel object recognition memory test is an additional procedure to describe and evaluate the memory of the rodents. The entire methodology was performed in a specific designed box of dimensions about $25 \mathrm{~cm} \times 25 \mathrm{~cm} \times 25 \mathrm{~cm}$ at daytime $\left(25 \pm 2^{\circ} \mathrm{C}\right)$. It was composed of quarter phases: (1) prehabituation, (2) habituation, (3) training, and (4) testing. All the mice that need to be tested were brought inside the testing room 30-35 min earlier than the commencement of the trial on the $1^{\text {st }}$ day, to get familiarized with the surroundings. They were kept to freely explore the testing box with objects for a $5 \mathrm{~min}$ period [20]. The rodents were habituated inside the box for $20 \mathrm{~min}$ on days 2 and 3. On day 4 , each mouse was brought out to a trial of training accompanied by a trial of testing. In the training trial, 2 objects were located oppositely to each other inside the box at a similar space from the adjacent corner [21]. The rodents were allowed to observe the objects for $10 \mathrm{~min}$ and were carried back to their home cages. Subsequently, they were put back to the similar specific investigational box, while this time, one of the two recognized objects was exchanged by a novel/new object [22]. All the performances were videotaped with a camera, and the recognition index was computed with the following formula: recognition test $=[$ time spent with the novel object/time spent with object $1+$ novel object $] \times 100$.

2.9. Statistical Analysis. All numerical values were expressed as mean \pm SEM. One-way ANOVA was used to express the results statistically, and the value of significance was set at $p<0.05$.

\section{Results}

This study reported a series of thirteen newly synthesized biaryl guanidine (fluorinated heteroaromatic side chain substituted) ligands intended to inhibit $\beta$-secretase enzymes. Among them, 1,3-bis(2-fluorophenyl)guanidine (1); 1,3bis(4-bromonaphthalen-1-yl)guanidine (2); 1,3-bis(2-nitrophenyl)guanidine (3); 1,3-di(pyridin-2-yl)guanidine (4); 1,3-di(quinolin-6-yl)guanidine (5); 1,3-bis(4-fluoronaphthalen-2-yl)guanidine (6); 1,3-di(naphthalen-2-yl)guanidine (7); 1,3-bis(3,5-bis(trifluoromethyl)phenyl)guanidine (8); 1,3-bis(5,6-difluoropyridin-3-yl)guanidine (9); 1,3-bis(4bromophenyl)guanidine (10); 1,3-bis(4-chloro-2-fluorophenyl)guanidine (11); 1,3-bis(4-fluorophenyl)guanidine (12); and 1,3-bis(3,4-difluorophenyl)guanidine (13) were enlisted. The entire series of the ligands was docked into the active catalyzing pocket of BACE1. Selected docking results were examined, and the maximum values of the compounds fill generally the active catalyzing domain of the dyadic aspartate within the flap region of the BACE1 enzyme. Figure 1 describes in detail the interaction of the best novel compounds (5), (8), and (9) within the active domain of the flap region of the BACE1 in closed conformation. They were found to inhibit the catalytic activity of the aspartate residues of this enzyme for intended interventions of $\mathrm{AD}$. Among all the synthesized ligands, compound (9) was found to pose $99 \%$ BACE1 inhibitory potential $\left(\mathrm{IC}_{50}=97 \pm 0.91 \mathrm{nM}\right)$ within the active pocket of the two key aspartic acids at 500 pmol having 


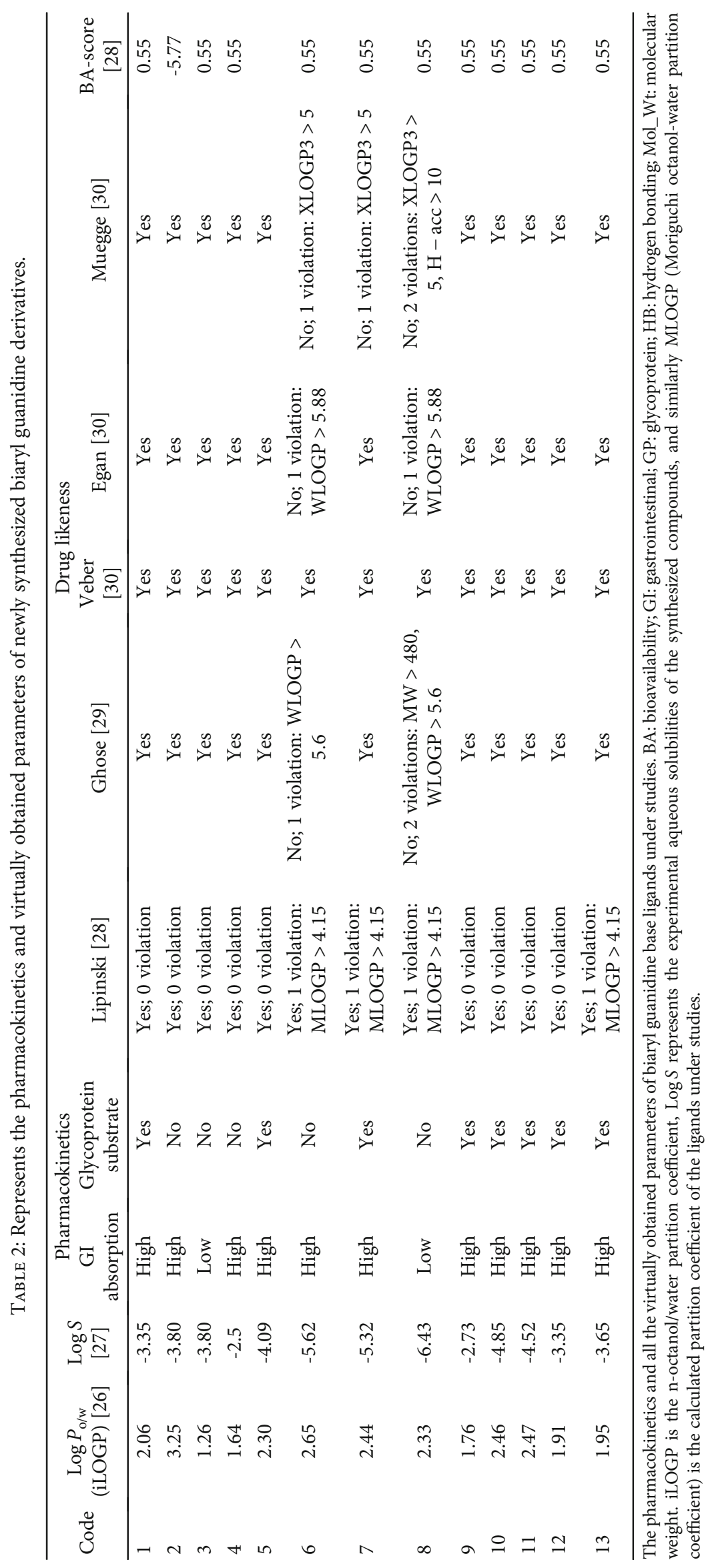



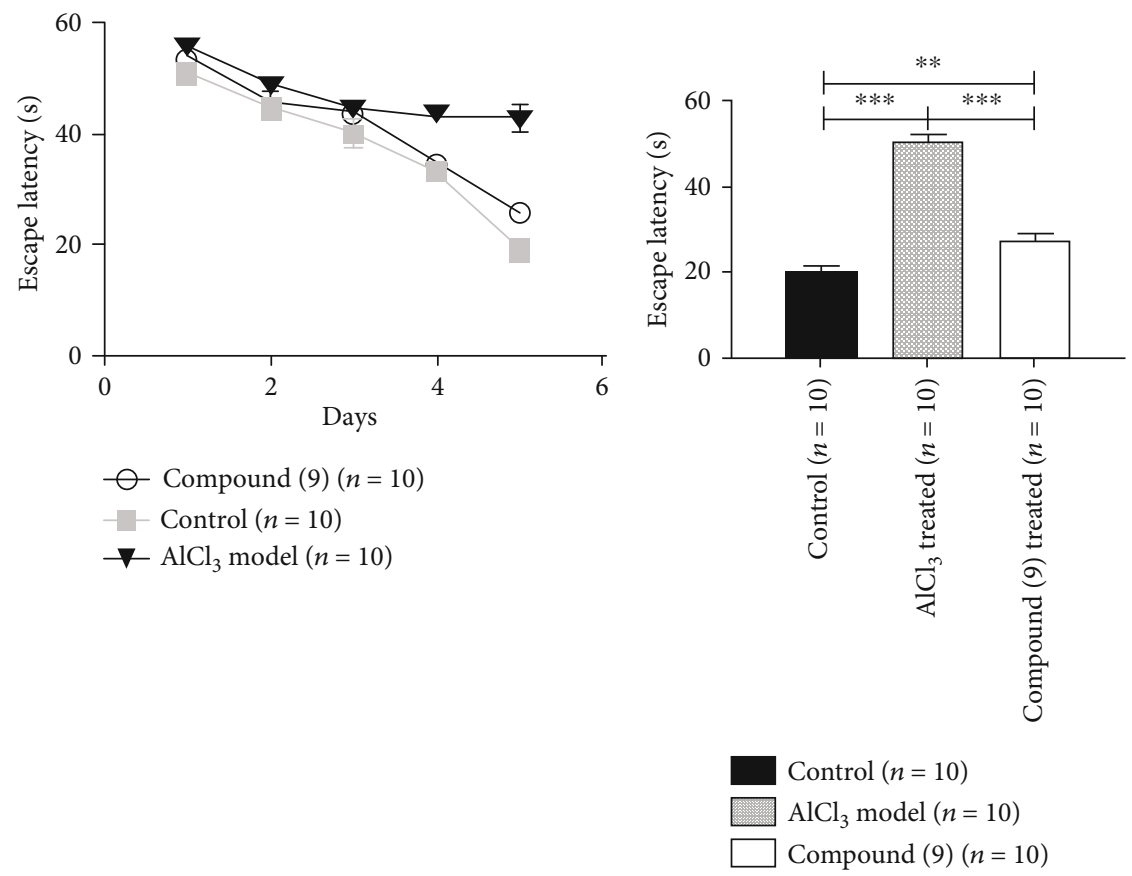

(a)

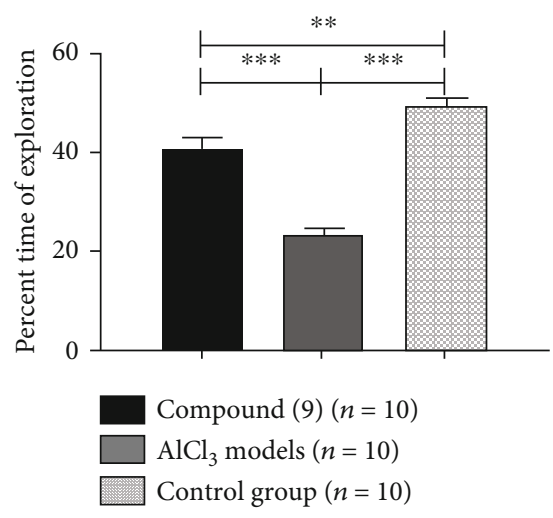

(c) (b)

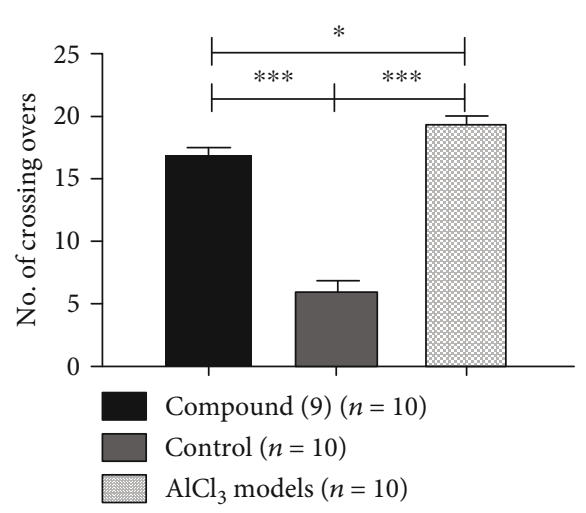

(d)

Figure 2: The synthesized compound (9) on the memory/learning behaviors of the mouse models manipulating the available technique of Morris water maze assay. (a) shows the escape latency time period of the mice to arrive at the platform on various trial days of the $\mathrm{AlCl}_{3}$ triggered neurotoxicity group, the control group, and the compound (9)-treated group. (b) signifies the analysis results among the three groups in terms of escape latency on the test day. (c) establishes the per hundred total time spent of the mice in the investigations of the target quadrant. (d) demonstrates in what manner each time every mouse crisscrossed the targeted quadrant.

the optimum values of all parameters. Compound (10) was found the least effective (35\% BACE1 inhibition with $\mathrm{IC}_{50}=$ $321 \pm 2.15 \mu \mathrm{M})$ while the rest of was found in between the highest (9) and the lowest (10) effective compounds. Table 1 describes in detail chemical structures, spectroscopic analysis, $\mathrm{IC}_{50}$, and some important physical/chemical properties while Table 2 summarizes pharmacokinetics and virtually obtained parameters (Figures 1S to 24Srepresented NMR spectra of the newly synthesized compounds available as supplementary material).

The in vivo assay "Morris water maze test" explained the aluminum chloride-induced neurotoxicity which is shown by an increase in time of escape latency $(50.60 \pm 3.70)$ compared to the control group $(20.20 \pm 2.01)$ of mice at day 5 . Compound (9) significantly improved the graphical record along with behavior of spatial learning and recalling power $(27.72 \pm 2.60 ; p<0.05)$ when compared with $\mathrm{AlCl}_{3}$-induced neurotoxic mice; similarly, an extra time was utilized in exploration of the targeted quadrant $(49.60 \pm 2.35)$ while the $\mathrm{AD}$-induced rodents showed23.6 \pm 2.01 and the treated mice showed a quite better interest in the quadrant lying a stand which on average is $41.07 \pm 2.5$. The AD model crisscrossed the respective quadrant $(6.10 \pm 1.0)$ while the control group spanned quite frequently $(19.80 \pm 1.90)$. Moreover, the compound (9)-treated group of mice presented the results within 2 designated groups $(16.70 \pm 1.07)$ Figure 2. 


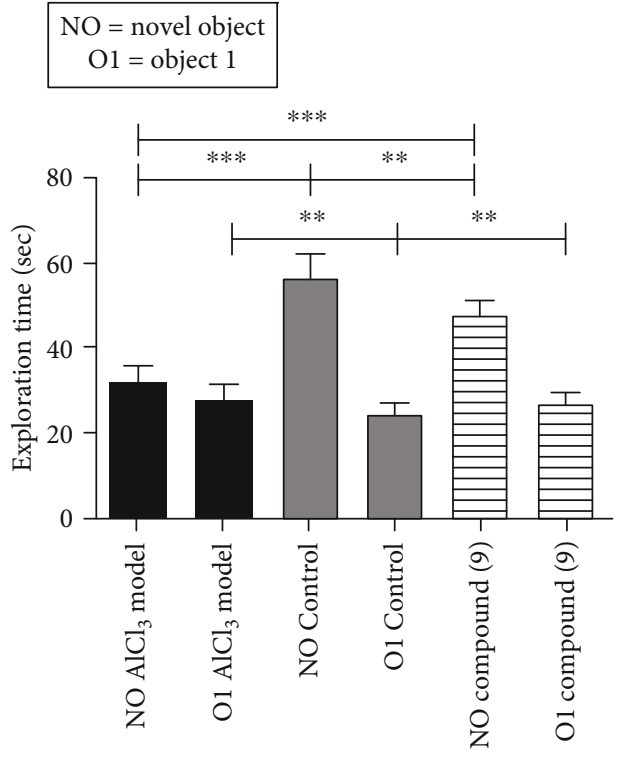

(a)

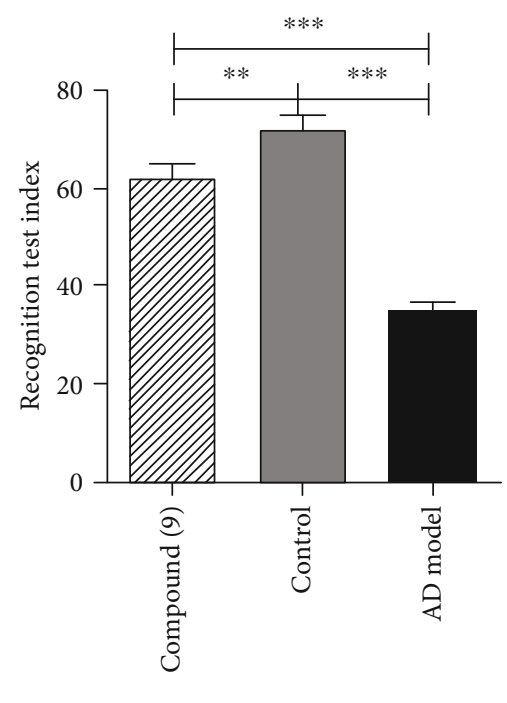

(b)

Figure 3: Compound (9) activities have stopped the decline of the 3-D spatial learning/memory of $\mathrm{AlCl}_{3}$-induced mouse models at some stage. The average times of explorations and the recognition indices are indicated significant with a $p<0.0002$ in both cases (one-way ANOVA test). The $f$-ratio value is 10.80 ; all the results are significant at $p<0.05$; error bars represent mean \pm SEM; the ( $* *$ ) shows less difference while $(* * *)$ shows a statistical significant difference.

The novel/new object recognition test showed insignificant variations in the time spent by the rodents in the initial training session. On the testing day, the recognition test index (RTI) was found to be $17.8 \%, 23.2 \%$, and $62.9 \%$, respectively, for the control, $\mathrm{AD}$ model, and $\mathrm{AD}$ modeltreated mice with our ligand "compound $(9)$ " $(p<0.05)$ as shown in Figure 3. The notable exploration time for the control $(64.44 \pm 2.50 \mathrm{~s}), \mathrm{AD}$ model $(31.80 \pm 2.00 \mathrm{~s})$, and $\mathrm{AD}$ model treated with compound (9) $(53.80 \pm 2.15 \mathrm{~s})(p<0.05$ ) was also compared (Figure 2). The exploration time and RTI which were the average times of explorations and the recognition indices have shown critical differences with a $p$ $<0.0002$ in both cases (one-way ANOVA test). The $f$-ratio value is 10.80; all the results are significant at $p<0.05$. Newly synthesized compound (9) was found to have stopped the decline of the recognition power of the mice as represented by the results obtained from the in vivo assays which were perfectly parallel with our in vitro outcomes which were in turn in accordance with the in silico results for the BACE1 inhibitory activity.

\section{Discussion}

Aspartic protease BACE1 ( $\beta$-secretase) catalysis is a ratelimiting step for conversion of APP into A $\beta 42$ that aggregated and led to senile plaque formation which is a wellknown pathogenesis of $\mathrm{AD}[1,21]$. The combined effects strengthened the amyloid cascade hypothesis, therefore targeting BACE1 as a therapeutic strategy to stop the progression of $\mathrm{AD}$. According to the literature survey, a lot of work has been done to come up with computationally designed, virtually screened, and chemically synthesized potent BACE1 inhibitors; however, a dire need has been felt recently to develop novel inhibitors to arrest $\beta$-secretase activity [22]. The present work predicted thirteen ligands as active through virtual screening, which eventually were synthesized as new compounds against $\beta$-secretase; therefore, compound (9) was found to have better inhibitory activity; it has fluorines attached directly to the side chains of the heteroaromatic ring of the guanidine nucleus [21]. Fluorine attachment imparted remarkable BACE1 inhibitory activity, besides an improved bioavailability at the active domain of the flap region of the BACE1 enzyme. Virtual screening further predicted that the ligands with binary aryl side chains were linked with a wide array of BACE1 inhibitory potentials. Any variation in fluorine moiety positions was found to have resulted in a drastic shift in attachment within the flap region in a closed conformation of BACE1 enzymes [22, 23]. It was revealed that fluorine atoms on the pyridine ring imparted enhanced efficiency (99\% BACE1 inhibition; $\left.\mathrm{IC}_{50}=97 \pm 0.91 \mathrm{nM}\right)$ and better bioavailability to arrest harmful effects at the specified target domain of BACE1 enzymes.

The Morris water maze behavioral test was employed to analyze the spatial memory in mice and remained valuable to examine the deficits in performances with $\mathrm{AD}$ animal models. However, strict infectious agent-free environment is a complex practice and often poses stress to the mice, so this test was also accompanied with another behavioral assay "novel object recognition assay." The novel object recognition test is based on the acquired instinct of animals to explore the novelty $[24,25]$. Moreover, this test did not need any positive or negative stimulus which sometimes negatively affects the results. On the account of all physiologic relevance, and to balance the results of MWMA if there is any deficiency, the NORT has also been carried out $[23,24]$. 


\section{Data Availability}

The present data regarding synthesis of compounds was generated in the laboratory of Prof. Dr. Borhan Babak (Department of Chemistry, Michigan State University, East Lansing, Michigan 48824, USA) and would be available on demand from the mentor. However, animal studies and molecular docking were conducted at the Department of Pharmacy, COMSATS University Islamabad, Pakistan, after getting permission from the Departmental Review Committee (Ref. \# PHM.Eth/CS-M01/18-001 dated 05/2018). However, FRET assays were performed at Rizvanov's laboratory (KFU, Russia).

\section{Conflicts of Interest}

Authors have found no competing interest.

\section{Authors' Contributions}

SA, FK, and GM designed the scheme and synthesized the proposed medicinal compounds. JI, AAR, and IH performed the virtual screening and FRET assays. MHHBA performed the experiments on laboratory animals and wrote the entire manuscript, while BB critically analyzed the whole data and supervised collectively.

\section{Acknowledgments}

This research work was partially supported by the COMSATS University Islamabad, Abbottabad Campus, under the funding project \#16-60/CRGP/CIIT/ABT/14/635. Authors wish to acknowledge Higher Education Commission (HEC) of Pakistan for its valuable support to accomplish this work. Moreover, authors are thankful to Dr. Asad MHHB (CUI, Pakistan; KFU, Russia), Prof. Dr. Rizvanov AA (KFU, Russia), and Prof. Dr. Iqbal J (CUI, Pakistan) for their efforts to finalize this work.

\section{Supplementary Materials}

Figure 1S: ${ }^{1} \mathrm{H}-\mathrm{NMR}$ spectrum of compound (1). Figure $2 \mathrm{~S}$ : ${ }^{13} \mathrm{C}$-NMR spectrum of compound (1). Figure $3 \mathrm{~S}:{ }^{19} \mathrm{~F}$-NMR spectrum of compound (1). Figure 4S: ${ }^{1} \mathrm{H}-\mathrm{NMR}$ spectrum of compound (2). Figure 5S: ${ }^{1} \mathrm{H}-\mathrm{NMR}$ spectrum of compound (3). Figure $6 \mathrm{~S}:{ }^{13} \mathrm{C}-\mathrm{NMR}$ spectrum of compound (3). Figure 7S: ${ }^{1} \mathrm{H}-\mathrm{NMR}$ spectrum of compound (4). Figure 8S: ${ }^{1} \mathrm{H}$-NMR spectrum of compound (5). Figure 9S: ${ }^{1} \mathrm{H}-\mathrm{NMR}$ spectrum of compound (6). Figure 10S: ${ }^{19}$ F-NMR spectrum of compound (6). Figure 11S: ${ }^{1} \mathrm{H}-\mathrm{NMR}$ spectrum of compound (7). Figure 12S: ${ }^{13} \mathrm{C}$-NMR spectrum of compound (7). Figure 13S: ${ }^{1} \mathrm{H}-\mathrm{NMR}$ spectrum of compound (8). Figure 14S: ${ }^{19} \mathrm{~F}-\mathrm{NMR}$ spectrum of compound (8). Figure $15 \mathrm{~S}:{ }^{1} \mathrm{H}-$ NMR spectrum of compound (9). Figure 16S: ${ }^{13} \mathrm{C}-\mathrm{NMR}$ spectrum of compound (9). Figure 17S: ${ }^{1} \mathrm{H}-\mathrm{NMR}$ spectrum of compound (10). Figure $18 \mathrm{~S}:{ }^{13} \mathrm{C}-\mathrm{NMR}$ spectrum of compound (10). Figure 19S: ${ }^{1} \mathrm{H}-\mathrm{NMR}$ spectrum of compound (11). Figure 20S: ${ }^{1} \mathrm{H}-\mathrm{NMR}$ spectrum of compound (12). Figure 21S: ${ }^{19} \mathrm{~F}-\mathrm{NMR}$ spectrum of compound (12). Figure 22S: ${ }^{1} \mathrm{H}-\mathrm{NMR}$ spectrum of compound (13). Figure $23 \mathrm{~S}:{ }^{13} \mathrm{C}-$
NMR spectrum of compound (13). Figure $24 \mathrm{~S}:{ }^{19} \mathrm{~F}-\mathrm{NMR}$ spectrum of compound (13). (Supplementary Materials)

\section{References}

[1] J. A. Hardy and G. A. Higgins, "Alzheimer's disease: the amyloid cascade hypothesis,” Science, vol. 256, no. 5054, pp. 184$185,1992$.

[2] R. Vassar, "The beta-secretase, BACE: a prime drug target for Alzheimer's disease," Journal of Molecular Neuroscience, vol. 17, no. 2, pp. 157-170, 2001.

[3] D. J. Selkoe, "The molecular pathology of Alzheimer's disease," Neuron, vol. 6, no. 4, pp. 487-498, 1991.

[4] M. Citron, "Alzheimer's disease: strategies for disease modification," Nature Reviews Drug Discovery, vol. 9, no. 5, pp. 387-398, 2010.

[5] R. Yan, M. J. Bienkowski, M. E. Shuck et al., "Membraneanchored aspartyl protease with Alzheimer's disease betasecretase activity," Nature, vol. 402, no. 6761, pp. 533-537, 1999.

[6] S. Karch, J. Broichhagen, J. Schneider et al., "A new fluorogenic small-molecule labeling tool for surface diffusion analysis and advanced fluorescence imaging of $\beta$-site amyloid precursor protein-cleaving enzyme 1 based on silicone rhodamine: SiRBACE1," Journal of Medicinal Chemistry, vol. 61, no. 14, pp. 6121-6139, 2018.

[7] X. Lin, G. Koelsch, S. Wu, D. Downs, A. Dashti, and J. Tang, "Human aspartic protease memapsin 2 cleaves the betasecretase site of beta-amyloid precursor protein," Proceedings of the National Academy of Sciences of the United States of America, vol. 97, no. 4, pp. 1456-1460, 2000.

[8] P. Jain, P. K. Wadhwa, S. Rohilla, and H. R. Jadhav, "Rational design, synthesis and in vitro evaluation of allylidene hydrazinecarboximidamide derivatives as BACE-1 inhibitors," Bioorganic \& Medicinal Chemistry Letters, vol. 26, no. 1, pp. 33-37, 2016.

[9] C. G. Vazhappilly, E. Saleh, W. Ramadan et al., "Inhibition of SHP2 by new compounds induces differential effects on RAS/$\mathrm{RAF} / \mathrm{ERK}$ and PI3K/AKT pathways in different cancer cell types," Investigational New Drugs, vol. 37, no. 2, pp. 252 261, 2019.

[10] H. Li, K. S. Leung, M. H. Wong, and P. J. Ballester, "Improving AutoDock Vina using random forest: the growing accuracy of binding affinity prediction by the effective exploitation of larger data sets," Molecular Informatics, vol. 34, no. 2-3, pp. 115-126, 2015.

[11] T. Oleg and A. J. Olson, “AutoDock Vina: improving the speed and accuracy of docking with a new scoring function, efficient optimization, and multithreading," Journal of Computational Chemistry, vol. 31, pp. 455-461, 2009.

[12] H. Xing, Y. Zhang, Y. Lai, Y. Jiang, and D. Ma, "Synthesis of symmetrical and unsymmetrical $\mathrm{N}, \mathrm{N}^{\prime}$-diaryl guanidines via copper/N-methylglycine-catalyzed arylation of guanidine nitrate," Journal of Organic Chemistry, vol. 77, no. 12, pp. 5449-5453, 2012.

[13] O. René, A. Souverneva, S. R. Magnuson, and B. P. Fauber, "Efficient syntheses of 2-fluoroalkylbenzimidazoles and -benzothiazoles," Tetrahedron Letters, vol. 54, no. 3, pp. 201-204, 2013.

[14] R. Yan and R. Vassar, "Targeting the $\beta$ secretase BACE1 for Alzheimer's disease therapy," The Lancet Neurology, vol. 13, no. 3, pp. 319-329, 2014. 
[15] M. C. Maillard, M. E. Perlman, O. Amitay et al., "Design, synthesis, and pharmacological evaluation of conformationally constrained analogues of N, N '-diaryl-and N-aryl-N-aralkylguanidines as potent inhibitors of neuronal $\mathrm{Na}+$ channels," Journal of Medicinal Chemistry, vol. 41, no. 16, pp. 30483061, 1998.

[16] C. X. Zhang, G. J. Zheng, F. Q. Bi, and Y. L. Li, "A simple and efficient synthesis of the valsartan," Chinese Chemical Letters, vol. 19, no. 7, pp. 759-761, 2008.

[17] P. Bamborough, J. A. Christopher, G. J. Cutler et al., "5-(1HBenzimidazol-1-yl)-3-alkoxy-2-thiophenecarbonitriles as potent, selective, inhibitors of IKK-epsilon kinase," Bioorganic \& Medicinal Chemistry Letters, vol. 16, no. 24, pp. 6236-6240, 2006.

[18] C. P. Hutton, J. A. Lemon, B. Sakic et al., "Early intervention with a multi-ingredient dietary supplement improves mood and spatial memory in a triple transgenic mouse model of Alzheimer's disease," Journal of Alzheimer's Disease, vol. 64, no. 3 , pp. 835-857, 2018.

[19] A. Gregory, Amyloid beta 4-42 in Alzheimer's disease: target, therapy, mechanism, [Ph.D. thesis], Georg-August University Göttingen, Germany, 2016.

[20] J. Cheng, P. M. Giguere, C. M. Schmerberg et al., "Further advances in optimizing (2-phenylcyclopropyl) methylamines as novel serotonin 2C agonists: effects on hyperlocomotion, prepulse inhibition, and cognition models," Journal of Medicinal Chemistry, vol. 59, no. 2, pp. 578-591, 2016.

[21] Schizophrenia Working Group of the Psychiatric Genomics Consortium, "Biological insights from 108 schizophreniaassociated genetic loci," Nature, vol. 511, no. 7510, pp. 421427, 2014.

[22] W. J. Thomsen, A. J. Grottick, F. Menzaghi et al., "Lorcaserin, a novel selective human 5-hydroxytryptamine2C agonist: in vitro and in vivo pharmacological characterization," Journal of Pharmacology and Experimental Therapeutics, vol. 325, no. 2, pp. 577-587, 2008.

[23] D. Oehlrich, H. Prokopcova, and H. J. M. Gijsen, "The evolution of amidine-based brain penetrant BACE1 inhibitors," Bioorganic \& Medicinal Chemistry Letters, vol. 24, no. 9, pp. 2033-2045, 2014.

[24] M. Citron, " $\beta$-secretase as a target for the treatment of Alzheimer's disease," Journal of Neuroscience Research, vol. 70, no. 3, pp. 373-379, 2002.

[25] M. E. Kennedy, W. Wang, L. Song et al., "Measuring human beta-secretase (BACE1) activity using homogeneous timeresolved fluorescence," Analytical Biochemistry, vol. 319, no. 1, pp. 49-55, 2003.

[26] J. A. Platts, S. P. Oldfield, M. M. Reif, A. Palmucci, E. Gabano, and D. Osella, "The RP-HPLC measurement and QSPR analysis of $\log \mathrm{P}(\mathrm{o} / \mathrm{w})$ values of several $\mathrm{Pt}(\mathrm{II})$ complexes," Journal of Inorganic Biochemistry, vol. 100, no. 7, pp. 1199-1207, 2006.

[27] J. Ali, P. Camilleri, M. B. Brown, A. J. Hutt, and S. B. Kirton, "Revisiting the general solubility equation: in silico prediction of aqueous solubility incorporating the effect of topographical polar surface area," Journal of Chemical Information and Modeling, vol. 52, no. 2, pp. 420-428, 2012.

[28] C. A. Lipinski, F. Lombardo, B. W. Dominy, and P. J. Feeney, "Experimental and computational approaches to estimate solubility and permeability in drug discovery and development settings," Advanced Drug Delivery Reviews, vol. 23, no. 1-3, pp. 3-25, 1997.
[29] T. K. Ghose, "Measurement of cellulase activities," Pure and Applied Chemistry, vol. 59, no. 2, pp. 257-268, 1987.

[30] D. F. Veber, S. R. Johnson, H. Y. Cheng, B. R. Smith, K. W. Ward, and K. D. Kopple, "Molecular properties that influence the oral bioavailability of drug candidates," Journal of Medicinal Chemistry, vol. 45, no. 12, pp. 2615-2623, 2002. 